\title{
Packetostatics: Deployment of Massively Dense Sensor Networks as an Electrostatics Problem
}

\author{
S. Toumpis \\ Telecommunications Research Center Vienna (ftw.) \\ Donau-City-Straße 1/3, A-1220, Vienna, Austria \\ toumpis@ftw.at
}

\author{
L. Tassiulas \\ Computer Engineering and Telecommunications Department \\ University of Thessaly, Glavani 37, Volos, 38221, Greece \\ leandros@inf.uth.gr
}

\begin{abstract}
We investigate the spatial distribution of wireless nodes that can transport a given volume of traffic in a sensor network, while requiring the minimum number of wireless nodes. The traffic is created at a spatially distributed set of sources, and must arrive at a spatially distributed set of sinks. Under a general assumption on the physical and medium access control (MAC) layers, the optimal distribution of nodes induces a traffic flow identical to the electrostatic field that would exist if the sources and sinks of traffic were substituted with an appropriate distribution of electric charge.

This analogy between Electrostatics and wireless sensor networks can be extended in a number of different ways. For example, Thomson's theorem on the distribution of electric charge on conductors gives the optimal distribution of traffic sources and sinks (that minimizes the number of nodes needed) when we have a limited degree of freedom on their initial placement. Electrostatics problems with Neumann boundary conditions and topologies with different types of dielectric materials can also be interpreted in the context of wireless sensor networks.

The analogy also has important limitations. For example, if we move to a three dimensional topology, adapting our general assumption on the physical and MAC layers accordingly, or we stay in the two dimensional plane but use an alternative assumption, that is more suited to Ultra WideBand communication, the optimal traffic distribution is not in general irrotational, and so can not be interpreted as an electrostatic field. Finally, the analogy can not be extended to include networks that support more than one type of traffic.

Keywords: Electrostatics, Neumann's boundary conditions, node placement, sensor networks, potential fields, sensor deployment, sensor networks, Thomson's theorem, wireless ad hoc networks.
\end{abstract}

\section{INTRODUCTION}

\section{A. Wireless Sensor Networks}

Wireless sensor networks appear at the intersection of three different technological areas: wireless communications, sensing technology, and networking. They consist of sensors that are equipped with wireless transceivers, and so are able to form a network. The sensors use this network in order to coordinate their sensing activities, and thus enhance their sensing capabilities, but also in order to send the information they collect to a central location [1].

The commercial deployment of wireless sensor networks is only now being made feasible, by continuous advances in hardware design, and there are still significant design

Work supported by Kplus funding for the ftw. project I0 "Signal and Information Processing", by the European Network of Excellence NEWCOM, and by the ARO grant W911NF-04-1-0306. issues that remain unresolved. This fact, coupled with the enormous commercial interest for sensor networks, make the area very attractive to researchers. Indeed, the research community is active on many different fronts, for example on the development of appropriate physical layer protocols [2], the calculation of their information theoretic capacity [3], the design of Medium Access Control (MAC) protocols [4], [5] and routing protocols [6], and the application layer [7].

Certain critical aspects of sensor networks are not shared by other types of wireless networks, therefore this setting is in many ways unique. As an example, the vast majority of sensor networks under design will be required to stay operational for large periods of time, on the order of many years, with batteries as their only energy source. Consequently, there has been significant research interest in the design of protocols that are extremely energy efficient [1], [8].

As another example, in most envisioned applications, it is expected that many more sensors than the necessary amount will be scattered in the covered area. Network designers can take advantage of this fact in order to maximize the lifetime of the network, for example by having some of the nodes stay in an energy conserving sleep mode [9].

Finally, in many applications the data collected by neighboring sensors are correlated. (This is the case, for example, in network designed to measure temperatures.) The designers can take advantage of this correlation to perform joint signal processing and compression, and therefore drastically reduce the volume of data that must be transported to the central data collector [10], [11].

\section{B. Overview of contributions}

In this work we investigate a setting that, to the best of our knowledge, has not attracted significant research interest until now. As shown in Fig. 1, we consider an environment in which there are a spatially distributed set of traffic sources and a spatially distributed set of traffic sinks, and a large number of wireless nodes, to be used for the transport of data from the sources to the sinks. The spatial distribution of the sources and the sinks is fixed, but we are free to place the wireless nodes as we like. We are interested in calculating the minimum number of nodes needed to support the traffic, and the associated placement of nodes that achieves this minimum. In other words, we are given a task (the transport of information from the sources to the sinks) and a set of resources (the wireless 


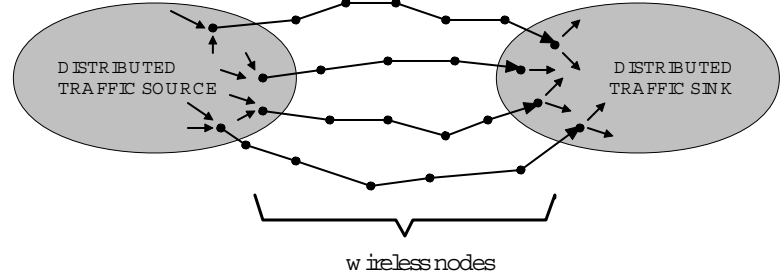

Fig. 1. Our setting: A set of wireless nodes are deployed in an area to support the transfer of information from a distributed traffic source to a distributed traffic sink.

nodes), and we would like to determine what is the minimum of resources needed, and how this minimum of resources should be deployed to achieve the task at hand.

We make a number of important assumptions, that we now list. First, we assume that we have so many nodes, that it does not make sense to describe their deployment in terms of the placement of individual nodes, but rather in terms of the node density at each location, measured in nodes per square meter. Following [12], we describe such networks as massively dense. As many sensor networks that are currently on the design phase are expected to consist of tens of thousands of nodes [1], this assumption is very reasonable. Therefore, sensor networks consisting of only a few nodes are out of this work's scope.

Secondly, we are not interested in finding the distribution of nodes that leads to an efficient harvest of the information from the traffic sources (this important problem is addressed, for example, in [13]). Rather, we assume the existence of a primary set of sensors, collocated with the traffic sources, whose only responsibility is the efficient collection of the created information, and we are interested in finding the distribution of a secondary set of wireless nodes, whose sole responsibility is the transport of information from the primary set of sensors to the traffic sinks. The problem of optimally placing a set of nodes that perform both the harvesting and the transport of the data goes beyond the scope of this work.

In this setting, and under a general assumption on the physical and medium access control (MAC) layers, which we outline in Section II, we show in Section III that the optimal spatial distribution of wireless nodes induces a traffic pattern that is identical to the electrostatic field that would be induced if we substituted the traffic sources with a distribution of positive electric charge and the traffic sinks by a distribution of negative charge. We prove this analogy by showing that both fields (the electrostatic field and the traffic flow) must satisfy the same set of equations. Our general assumption on the physical and MAC layers comes naturally in many cases where the bandwidth available to the network is limited.

The analogy between Electrostatics and wireless sensor networks can be extended in various ways, some of which are explored in Section IV. For example, a well known fact in Electrostatics is Thomson's theorem, which states that when electric charges are infused in a set of conductors, the charges will be distributed on the surfaces of the conductors (by virtue of the electrostatic forces between them), so as to minimize the energy of the induced electric field. This theorem readily translates to our setting. As shown in Section IV-A, it implies that when the initial distribution of traffic sources and sinks is not given, but rather we are free to place sources and sinks within certain areas in space, then the placement that minimizes the number of nodes needed to support the flow of information induces a traffic pattern identical to the electrostatic field created in a corresponding Electrostatics topology, in which the areas where the traffic sources and sinks can be placed are replaced with conductors, infused with the appropriate amounts of electric charge.

As another example, many sensor networks are required to operate in environments that impose hard constraints on where nodes may be placed. As we show in Section IV-B, the optimal placement of nodes under these constraints induces a traffic flow identical to the electrostatic field in a topology that contains regions on which we impose Neumann boundary conditions.

It is often the case that the communication capabilities of the nodes depend on their placement on their environment. As an example, certain areas may be experiencing particularly high levels of interference, or may have heavy vegetation that absorbs the power of transmitted signals more than other areas. In Section IV-C we show that the problem of finding the optimal distribution of nodes in such an environment can be cast as the problem of finding the electric field in an Electrostatics topology that is comprised of different types of dielectrics.

On the other hand, our analogy between Electrostatics and wireless sensor networks has important limitations. For example, as we discuss in Section $\mathrm{V}$-A, if we move to a three dimensional topology, and adapt our general assumption on the physical and MAC layers accordingly, the optimal placement of nodes induces a traffic pattern that is no longer irrotational, and hence is not related to an electrostatic field.

In Section V-B we show that if we use another assumption on the physical and MAC layers, that is more appropriate when the communication is power limited, or equivalently the available bandwidth is very large [14], the optimal node distribution induces a traffic pattern that in general is not irrotational.

Finally, as we elaborate on Section V-C, the analogy hinges on the assumption that there is only one type of traffic, allowing us to map the sources of this traffic to positive charges, and the sinks of the traffic to negative charges. To handle two or more distinct types of traffic, we would require the existence of two or more distinct types of charges. Unfortunately, nature is not prepared to grant us this extra favor.

\section{Related Work}

The use of Electromagnetics in sensor network research has already appeared in the past. For example, in [15], the deployment of a network of wireless sensors with locomotion 
capabilities is considered. The authors propose a totally scalable and distributed protocol that leads to the maximization of the area that the sensor network covers. The protocol is based on the use of a virtual potential field, similar to an electrostatic potential field, which is constructed so that each node is repelled by both obstacles and other nodes, thereby forcing the network to spreads itself throughout the environment.

More recently, the author of [12] studied massively dense networks, that consist of so many nodes, that it is best to describe the network in terms of macroscopic quantities such as the node density and the volume of traffic at each point in space. In such a setting, and assuming that the volume of traffic varies in space, it is shown that the minimum-hop route between two locations in the network satisfies the same laws that govern the shape of rays of light in media with a spatially varying index of refraction.

After the bulk of our work was completed, we discovered that an application of Electrostatics somehow similar to ours has recently been reported in [16], [17]. In that work, a form of routing for wireless ad hoc networks is proposed that routes traffic along the lines of an appropriate electrostatic field. The justification for this type of routing is intuitive, whereas in our setting, which is not identical but somehow similar, we show that routing traffic along the line of an electrostatic field is optimal in some cases, in the sense that it minimizes the volume of resources needed, and suboptimal in other cases.

Finally, in [18], a multipath routing protocol is proposed, that routes the packets from their source to their destination along different lines of the electrostatic field that is created if we place a positive singular charge at the source and a negative singular charge at the destination. Contrary to our work, this work places the emphasis on the development of a practical and efficient routing protocol, and not on the identification of any theoretical analogy between electrostatics and networking.

\section{NETWORK MODEL}

In this section we first introduce three macroscopic quantities: the information density function $\rho(x, y)$, the node density function $d(x, y)$, and the traffic flow function $\mathbf{T}(x, y)^{1}$. We conclude by providing an equation linking the node density function and the maximum magnitude of the traffic flow function.

\section{A. Macroscopic Quantities}

We consider the unbounded two dimensional $x y$ plane, on which are placed distributed sources and sinks of information. We model the sources and sinks jointly, by the information density function $\rho(x, y)$, which is measured in $\mathrm{bps} / \mathrm{m}^{2}$. At locations $(x, y)$ where $\rho(x, y)>0$, there is a distributed traffic source, such that the rate with which information is generated within a surface of infinitesimal area $\epsilon$, centered at $(x, y)$, is $\epsilon \rho(x, y)$. At locations where $\rho(x, y)<0$, there is a distributed traffic sink, such that the required absorption rate within a surface of infinitesimal area $\epsilon$, centered at $(x, y)$, is $-\epsilon \rho(x, y)$.

\footnotetext{
${ }^{1}$ We denote scalars by lower case letters, and vectors by bold capitals.
}

We require that the total rate with which traffic sinks must absorb data is the same as the total rate with which the data is created. This requirement translates into the equation

$$
\int \rho(x, y) d S=0
$$

where the surface integral is taken over the whole plane.

To facilitate the transfer of information from the sources to the sinks, we are given a large number of wireless nodes, that we are free to place anywhere on the two dimensional area. Because we assume the number of nodes to be very large, we will describe their placement not in terms of their individual positions which are microscopic quantities, but rather in terms of a macroscopic quantity, the node density $d(x, y)$, measured in nodes $/ \mathrm{m}^{2}$. The total number of nodes, $N$, is given by

$$
N=\int d(x, y) d S
$$

where, again, the surface integral is taken over the whole plane.

In networks, the flow of information is typically described in terms of the rate with which information arrives in individual nodes. However, in our setting, we have a massively dense network, in which the rate of arrival of information in a particular node is a microscopic quantity. In this setting, we can best model the flow of traffic in the network in terms of the traffic flow function $\mathbf{T}(x, y)$, which is a macroscopic quantity.

$\mathbf{T}(x, y)$ is a vector function (with an $\mathrm{x}$-component and a $\mathrm{y}$-component), whose magnitude is measured in bps $/ \mathrm{m}$. It is defined so that (i) its direction coincides with the direction of the flow of information at point $(x, y)$, and (ii) $\epsilon|\mathbf{T}(x, y)|$ equals $^{2}$ the rate with which information crosses a linear segment of incremental length $\epsilon$, that is centered on $(x, y)$, and is perpendicular to $\mathbf{T}(x, y)$.

When viewing a microscopic part of the network, one may observe many distinct streams of traffic, possibly along different directions. However, the fact that the traffic streams all carry the same type of packets, allows us to combine them by performing vector addition, and thus abstract the movement of data at the microscopic level by a macroscopic quantity, the traffic flow function.

\section{B. Relation between the node density and the traffic flow}

Since the nodes are placed on the network to facilitate the transfer of information, it is important to identify how much traffic can be carried through a location $(x, y)$ with a node density $d(x, y)$. We make the following fundamental assumption (which was first used, in a different context, in [12]):

Assumption 1: A location $(x, y)$, where the node density is $d(x, y)$, can support any traffic flow vector with a magnitude less or equal to a bound $|\boldsymbol{T}(x, y)|_{\max }$ which is proportional to the square root of the density:

$$
|\boldsymbol{T}(x, y)| \leq|\boldsymbol{T}(x, y)|_{\max }=K \sqrt{d(x, y)},
$$

\footnotetext{
${ }^{2}$ By $|\mathbf{x}|$ we denote the magnitude, or length of a vector $\mathbf{x}$.
} 


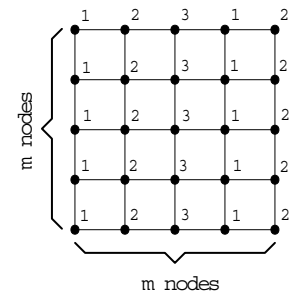

Fig. 2. A wireless network of $m^{2}$ nodes placed on a regular grid. Nodes connected by edges can interfere with each other. A time division scheme exists that allows each node to transmit a packet to the node directly on the right, in one out of every three slots.

where $K$ is a normalizing coefficient measured in bps.

The validity of Assumption 1 depends on the physical layer and the medium access control protocol used by the network. Although it is not generally true, it nonetheless holds in many different settings of interest.

As an example, let us consider the network of Fig. 2, in which $\mathrm{m}^{2}$ nodes are placed in a perfect square grid of $m \times m$ nodes. Regarding the channel model, it is assumed that each node can listen to transmissions from its four nearest neighbors (those at the edges only have two or three nearest neighbors), and the rest of the nodes can not be heard at all. We can model this channel by a graph, in which communicating nodes are connected by edges. This model is rather simplistic, however it retains certain critical aspects of the wireless channel, and therefore it is still widely used by the research community. Regarding the transceiver model, we assume that nodes transmit data with a fixed, global rate of $W$ bps, and transmissions are successful as long as no two data packets arrive at the same receiver at the same time.

In this network, it is possible for the nodes to use a simple time division that consists of only three slots, such that each node can transmit during exactly one of the three slots, to the node directly on the right. The slot that corresponds to each of the nodes appears in Fig. 2, next to the nodes.

Using this time division, the $\mathrm{m}^{2}$ nodes can be organized according to a scheme that will allow a traffic stream of size $T_{\text {local }}=\frac{m W}{3}$ bps to transverse the network from the left to the right. Indeed, we assume that the traffic enters the network in $m$ streams, each of size $\frac{W}{3}$, which are inserted in each of the $m$ nodes of the left-most column. As shown in Figure 3, the streams will be transmitted along straight lines, from left to right, each along $m$ nodes, and will terminate in the $m$ nodes of the right-most column. Since transmissions are with a rate $W$, and each node is transmitting during 1 out of every 3 slots, each stream of data will be of size $\frac{W}{3}$, bringing the total traffic transversing the network to $T_{\text {local }}=\frac{m W}{3}$ bps.

If we take the total area of the network to be $\epsilon^{2}$, the total number of nodes $m^{2}$ and the network density $d$ will satisfy the equation $m^{2}=\epsilon^{2} d$. Therefore, in terms of the node density $d$, the traffic crossing a line segment of length $\epsilon$ equals $T_{\text {local }}=$ $\epsilon \frac{W}{3} \sqrt{d}$. Clearly, no scheme exists that can achieve a larger traffic. As a result, Assumption 1 will hold for networks that

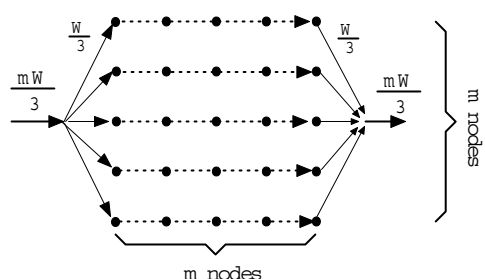

Fig. 3. A routing scheme under which the $m^{2}$ nodes of the network of Fig. 2 can support the transport of $T_{\text {local }}=\frac{m W}{3}$ bps through the network.

appear locally as the network of Fig. 2, with $K=\frac{W}{3}$.

The above setting could be described as simplistic, however the fact that a network of $m^{2}$ nodes can support a traffic on the order of $m$ also holds in more realistic settings. As another example, in [19] it was shown that the traffic that can be supported in the above network, if nodes access the channel by use of slotted Aloha instead of time division, is $T_{\text {local }}=k \times W \times m$, where $k$ is a constant, smaller than $\frac{1}{3}$, that captures the efficiency of Aloha.

As a final example, in [20] it was shown that a network of $n$ randomly placed nodes can support an aggregate traffic on the order of $\sqrt{\frac{n}{\log n}}$, even under a more realistic interference model that accounts for interferences coming from arbitrarily distant nodes. The logarithm in the denominator appears due to the proving methodology of [20], and it has been recently shown [21] that it can be dispensed off, by use of percolation theory in the proofs.

The results of [19], [20] assume traffic between nodes in the network, and not traffic transversing the network, as in our setting. However, in the second case each packet must be transported over a distance equal to the diameter of the network, and in the first case over a (possibly random) distance which is of the same order. Therefore, it is straightforward to show that the above results can be used in our setting.

\section{AnAlogy With Electrostatics}

In this section we first show that in order for information to appear only at the traffic sources and to disappear only at the traffic sinks, it is necessary that the divergence of the traffic flow equals the information density function. We then show that, in order to minimize the number of nodes needed to transport the information, it is necessary that the traffic flow has a zero curl. Jointly, these results show the traffic flow function that minimizes the number of required nodes is the same as the electrostatic field created by a charge density function equal to the information density function. We then show that a function analogous to the potential function of Electrostatics may be defined in the context of wireless sensor networks, that can be used to determine the number of hops needed to travel between different locations in the network. We conclude by presenting two indicative examples.

\section{A. The divergence of the traffic flow function}

Let $A$ be a surface on the $x y$ plane, of arbitrary shape. We will denote its boundary curve by $\partial A$ and its total area by 
$|A|$. For information to be conserved, it is necessary that the rate with which information is created in the area is equal to the rate with which information is leaving the area through its boundary $\partial A$. In other words, the following equality must hold:

$$
\int_{A} \rho(x, y) d S=\oint_{\partial A}[\mathbf{T}(x, y) \cdot \hat{\mathbf{n}}] d l,
$$

where $\hat{\mathbf{n}}$ is the unit vector normal to the boundary curve $\partial A$ at the point $(x, y)$, and pointing outside $A$, and the integral on the right hand side is the path integral [22] of the function $[\mathbf{T}(x, y) \cdot \hat{\mathbf{n}}]$. This function represents the rate (measured in $\frac{\mathrm{bps}}{\mathrm{m}}$ ) with which information is leaving $A$ at the point $(x, y)$ at its boundary $\partial A$.

Equation (4) must hold for any surface $A$. Therefore, it will also hold for a sequence of surfaces $A_{k}$ that all include in their interior an arbitrary point $\left(x_{0}, y_{0}\right)$, and are such that $\left|A_{k}\right| \rightarrow 0$. Applying (4) for $A_{k}$ we have:

$$
\int_{A_{k}} \rho(x, y) d S=\oint_{\partial A_{k}}[\mathbf{T}(x, y) \cdot \hat{\mathbf{n}}] d l .
$$

Assuming that $\rho(x, y)$ is continuous at $\left(x_{0}, y_{0}\right)$, we have that $^{3}$

$$
\int_{A_{k}} \rho(x, y) d S=\rho\left(x_{0}, y_{0}\right) \times\left|A_{k}\right|+o\left(\left|A_{k}\right|\right) .
$$

Combining (5) and (6), and taking the limit with respect to $k$, we arrive at

$$
\rho\left(x_{0}, y_{0}\right)=\lim _{k \rightarrow \infty} \frac{1}{\left|A_{k}\right|} \oint_{\partial A_{k}}[\mathbf{T}(x, y) \cdot \hat{\mathbf{n}}] d l \triangleq \nabla \cdot \mathbf{T}\left(x_{0}, y_{0}\right) .
$$

The limit in (7) is defined as the divergence of the vector function $\mathbf{T}$, at the point $\left(x_{0}, y_{0}\right)$ [23]. The divergence of the traffic flow function measures the degree with which the traffic flow increases (when information in injected in the network) or decreases (when information is removed from the network) at the particular point $\left(x_{0}, y_{0}\right)$. In cartesian coordinates, the divergence is given by the formula $\nabla \cdot \mathbf{T}=\frac{\partial T_{x}}{\partial x}+\frac{\partial T_{y}}{\partial y}$, however its intuitive meaning can best be conveyed by the limit of (7), which is independent of the choice of the coordinate system. More information on the definition and physical meaning of the divergence function, and its generalization in the threedimensional space, can be found in any book on vector calculus, for example in [23], or Electromagnetics, for example in [24].

To summarize, we have showed that

$$
\nabla \cdot \mathbf{T}=\rho .
$$

\section{B. The curl of the traffic flow function}

Equation (8) is not enough to uniquely specify the traffic flow function. To show this, we note that there is an infinity of distinct functions whose divergence is zero everywhere. Such functions are called solenoidal [23], and each of them represents a distinct way with which a quantity of liquid can flow on a two dimensional infinite plane with no sources and

\footnotetext{
${ }^{3}$ We write $f(x)=o(x)$ to denote that $\lim _{x \rightarrow 0} \frac{f(x)}{x}=0$.
}

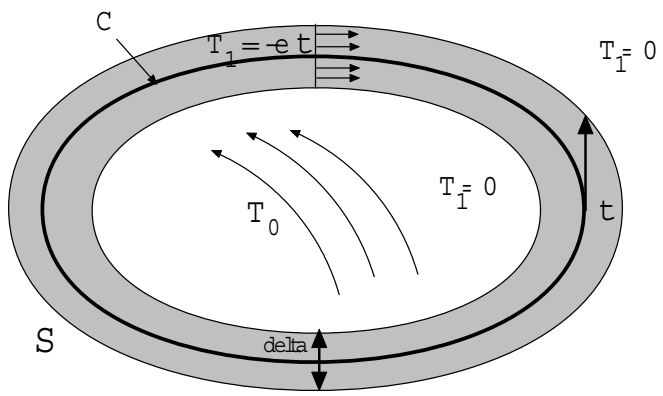

Fig. 4. The setup of the proof that the optimal traffic flow function has a zero curl.

sinks. Now let $\mathbf{T}_{0}$ be a flow that satisfies (8): $\nabla \cdot \mathbf{T}_{0}=\rho$. By the definition of the divergence (7), it follows that the divergence operator $\nabla \cdot$ is linear, therefore if $\nabla \cdot \mathbf{T}_{1}=0$, then $\nabla \cdot\left(\mathbf{T}_{0}+\mathbf{T}_{1}\right)=\rho$. Since there is an infinity of distinct solenoidal functions $\mathbf{T}_{1}$, the result follows.

We now show that among all traffic flow functions that satisfy (8), the one that needs the smallest number of nodes to be supported must also be irrotational, i.e. its curl must be zero everywhere:

$$
\nabla \times \mathbf{T}=0 .
$$

The curl $\nabla \times \mathbf{T}$ of a two dimensional vector $\mathbf{T}$ at a point $\left(x_{0}, y_{0}\right)$ is a scalar function defined as follows:

$$
\nabla \times \mathbf{T}=\lim _{\left|A_{k}\right| \rightarrow 0} \frac{\oint_{\partial A_{k}} \mathbf{T} \cdot d \mathbf{l}}{\left|A_{k}\right|},
$$

where $\left\{A_{k}\right\}$ is a sequence of surfaces of vanishing area, that contain $\left(x_{0}, y_{0}\right)$ in their interior, and the integral of the right hand side is the line integral [25] of the function $\mathbf{T}$ over the curve $\partial A_{k}$ (which is taken to have a counterclockwise direction). Intuitively, the magnitude of the curl at a point $\left(x_{0}, y_{0}\right)$ is a measure of how much circulation around the point $\left(x_{0}, y_{0}\right)$ the function $\mathbf{T}$ has. The circulation is counter-clockwise, if the curl is positive, and clockwise, if the circulation is negative. In cartesian coordinates, the curl of a function is given by $\nabla \times \mathbf{T}=\left(\frac{\partial \mathbf{T}_{y}}{\partial x}-\frac{\partial \mathbf{T}_{x}}{\partial y}\right)$. A more detailed exposition on curl, with its generalization in the three dimensions (which is a vector function) can be found in [23].

We now prove (9), by arriving at a contradiction. In particular, suppose that the traffic flow $\mathbf{T}_{0}$ that needs the minimum number of nodes has a non-zero curl at some point in space. It follows from (10) that there is a curve $\mathcal{C}$, of length $L$, along which the line integral of $\mathbf{T}_{0}$ is non-zero. By choosing a proper direction for $\mathcal{C}$, we can assume that the line integral is positive:

$$
\oint_{\mathcal{C}} \mathbf{T}_{0} \cdot d \mathbf{l}=p>0
$$

As shown in Fig. 4, we form around $\mathcal{C}$ a strip $S$ of infinitesimal and constant width $\delta$. Because $\delta$ is infinitesimally small, the area of the strip can be taken to be equal to $|S|=\delta \times L$.

We construct an auxiliary vector function $\mathbf{T}_{1}$ in the following manner: Outside the strip, $\mathbf{T}_{1}=\mathbf{0}$. Inside the strip, 
at a point $(x, y), \mathbf{T}_{1}=-\epsilon \mathbf{t}$, where $\mathbf{t}$ is a unit vector tangential to $\mathcal{C}$, at the point where $\mathcal{C}$ is closest to the point $(x, y)$. Therefore, we construct $\mathbf{T}_{1}$ to resemble the flow of a small quantity of liquid around a closed hose of impermeable boundaries, which goes against the average flow of $\mathbf{T}_{0}$ in $S$. By its physical interpretation, it is clear that $\mathbf{T}_{1}$ has a zero divergence everywhere. It is also straightforward to show this mathematically. Indeed, outside the strip $\mathbf{T}_{1}$ is identically zero, and inside the strip it can be shown that the divergence is zero by a direct application of the definition of (7).

As noted, the divergence operator is linear. Therefore:

$$
\nabla \cdot\left(\mathbf{T}_{0}+\mathbf{T}_{1}\right)=\nabla \cdot \mathbf{T}_{0}+\nabla \cdot \mathbf{T}_{1}=\rho,
$$

and it suffices to show that the traffic flow function $\left(\mathbf{T}_{0}+\mathbf{T}_{1}\right)$ can be supported by fewer nodes. Indeed, let $N_{0}$ be the total number of nodes needed to support $\mathbf{T}_{0}$ and $N_{0+1}$ be the total number of nodes needed to support $\mathbf{T}_{0}+\mathbf{T}_{1}$. We have:

$$
\begin{aligned}
N_{0} & -N_{0+1} \\
& =\int_{S}\left(\left|\mathbf{T}_{0}\right|^{2}-\left|\mathbf{T}_{0}+\mathbf{T}_{1}\right|^{2}\right) d S \\
& =\int_{S}\left(\left|\mathbf{T}_{0}\right|^{2}-\left|\mathbf{T}_{0}\right|^{2}-\left|\mathbf{T}_{1}\right|^{2}-2 \mathbf{T}_{0} \cdot \mathbf{T}_{1}\right) d S \\
& =-\int_{S} \epsilon^{2} d S+\int_{S} 2 \epsilon \mathbf{T}_{0} \cdot \mathbf{t} d S \\
& =-\epsilon^{2}|S|+2 \epsilon \delta \oint_{\mathcal{C}} \mathbf{T}_{0} \cdot d \mathbf{t}=-\epsilon^{2}|S|+2 \epsilon \delta p .
\end{aligned}
$$

The first equality comes from noting that the functions $\mathbf{T}_{0}$ and $\mathbf{T}_{0}+\mathbf{T}_{1}$ differ only within the surface $S$. The last one comes from applying (11). It follows that for a sufficiently small value of $\epsilon$, the traffic flow $\mathbf{T}_{0}+\mathbf{T}_{1}$ can be supported by a smaller number of nodes than the traffic flow $\mathbf{T}_{0}$. Therefore, we arrive at a contradiction, so (9) must hold ${ }^{4}$.

To summarize, we have proved that the traffic flow function must satisfy (8) and (9). These equations jointly do not uniquely specify the traffic flow. Indeed, provided there is a solution $\mathbf{T}_{0}$ that satisfies both of them, then so does $\mathbf{T}_{0}+\mathbf{c}$ where $\mathbf{c}$ is a constant vector. However, by Helmholtz's theorem [24], it follows that the solution exists and is uniquely specified if, in addition, we require that the traffic flow is zero at infinity:

$$
\left.\mathbf{T}\right|_{\infty}=\mathbf{0}
$$

Assuming that the traffic sources and sinks are constraint over a finite region, this is a reasonable boundary condition to take, as there is no need for the traffic flow to arrive at the sink by going first to infinity and back ${ }^{5}$.

To summarize, it follows by Helmholtz's theorem that (8), (9), (12) uniquely specify the traffic flow function. However, it is a basic fact of electrostatic field theory [24], that these

\footnotetext{
${ }^{4}$ Equation (9) can also be shown by use of calculus of variations, or straightforward vector equality manipulation, however these proofs are nonconstructive and therefore not as intuitive [26].

${ }^{5}$ Note that Helmholtz's theorem is typically mentioned in a three dimensional setting, however its two dimensional version follows as a special case.
}

equations also uniquely specify the electrostatic field $\mathbf{E}$ induced by a two-dimensional electric charge density $\rho(x, y)^{6}$. We are now ready to formally present our main result:

Theorem 1: In an environment with an information density function $\rho(x, y)$, in which Assumption 1 holds, the traffic density function $\boldsymbol{T}$ that minimizes the number of nodes needed to support $\rho(x, y)$ is uniquely specified by the equations

$$
\nabla \cdot \boldsymbol{T}=\rho, \quad \nabla \times \boldsymbol{T}=0,\left.\quad \boldsymbol{T}\right|_{\infty}=\boldsymbol{0} .
$$

Therefore, $\boldsymbol{T}$ is identical to the two-dimensional electrostatic field that would be induced by a two-dimensional density of electric charge equal to $\rho$.

The corresponding node density function $d(x, y)$ is given by

$$
d(x, y)=\frac{1}{K^{2}}|\boldsymbol{T}(x, y)|^{2},
$$

and the total number of nodes $N$ needed is given by

$$
N=\int \frac{1}{K^{2}}|\boldsymbol{T}(x, y)|^{2} d S
$$

where the surface integral is taken over the whole plane.

\section{The hop function}

It is a well known fact of vector calculus [23], [24] that an irrotational vector function $\mathbf{E}$ may be represented in terms of the gradient $\nabla U$ of a scalar function $U$ :

$$
\mathbf{E}=\nabla U \text {. }
$$

The difference $U(A)-U(B)$ is given by the line integral

$$
U(A)-U(B)=\int_{B}^{A} \mathbf{E} \cdot d \mathbf{l}
$$

where the line integral is along any curve that starts at $B$ and ends at $A$. When $\mathbf{E}$ is the electrostatic field, $-U$ is know as the potential function, and it follows from (15) that the difference $U(A)-U(B)$ is the amount of energy required to move a unit negative charge from point $B$ to point $A$.

Since, by Assumption 1, the traffic flow $\mathbf{T}$ is irrotational, it can also be associated with a function $H$ such that $\mathbf{T}=\nabla H$, which can also be shown to have a physical interpretation. In particular, consider a curve $\mathcal{C}$ along the trajectory of a packet stream, starting at a point $B$ and ending at a downstream point $A$. Equation (15) becomes:

$$
\begin{aligned}
& H(A)-H(B) \\
& \quad=\int_{B}^{A} \mathbf{T} \cdot d \mathbf{l}=\int_{B}^{A}|\mathbf{T}| d l=\int_{B}^{A} K \sqrt{d(x, y)} d l .
\end{aligned}
$$

The second equality comes from noting that, by its construction, curve $\mathcal{C}$ is parallel to $\mathbf{T}$. The third equality comes from assuming that Assumption 1 holds with equality, so that at each point the network uses its available resources optimally, following a scheme that resembles that of Fig. 3.

\footnotetext{
${ }^{6}$ By a two-dimensional density, we mean a density of charge immersed in the three dimensional space that is invariant with respect to the $z$ coordinate. By symmetry, the electric field it induces will also be invariant with respect to the $z$ coordinate, and with a zero $z$-component.
} 


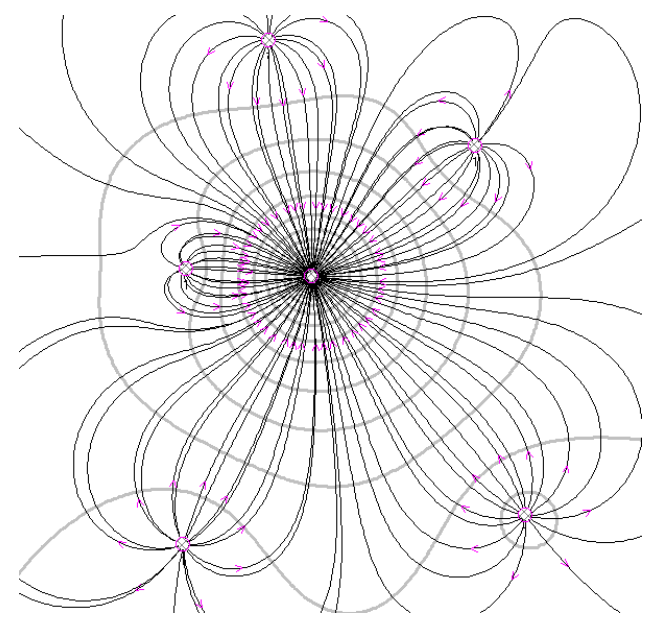

Fig. 5. Field lines (in thin black) and lines of constant potential (in thick gray), in a two dimensional topology consisting of 5 positive singular charges of equal magnitude, and a single singular negative charge of 5 times that magnitude.

As $\sqrt{d(x, y)} d l$ is the approximate number of hops that a packet needs to traverse an incremental length $d l$ at a point $(x, y)$ where the node density is $d(x, y)$, it follows that the difference $H(A)-H(B)$ is proportional to the number of hops needed for the packets to go from point $B$ to point $A$, when Assumption 1 holds with equality, and the network behaves locally as in Fig. 3. Because of its physical interpretation, we will refer to $H$ as the hop function.

\section{Examples}

Theorem 1 shows that a problem in wireless sensor networks, namely the minimization of the number of nodes needed to support a given traffic, is identical to a standard problem in Electrostatics, i.e., the calculation of the electrostatic field induced by a two-dimensional distribution of charge. As a result, we can use the large amount of knowledge, intuition, and solving techniques that have been accumulated over the last hundred years in the fields of Electrostatics and calculus of irrotational vector functions, in the context of wireless sensor networks.

As an example, let us consider the topology of Fig. 5, in which we have placed 5 positive singular charges of equal magnitude and a single singular negative charge of 5 times that magnitude, at the center of the setting. The induced electrostatic field can be calculated by using any of a large number of software tools that are available, either for solving arbitrary PDEs, or for solving Electrostatics problems in particular. In this, and the following examples, we use the specialized software tool of [27].

As is standard in Electrostatics, we denote the electric field by use of field lines. These are defined in the following manner: The field line crossing a point $(x, y)$ is parallel to $\mathbf{E}(x, y)$, and the density of field lines at that point is proportional to the magnitude $|\mathbf{E}(x, y)|$. In the figure we have also plotted lines of constant potential. As follows from the

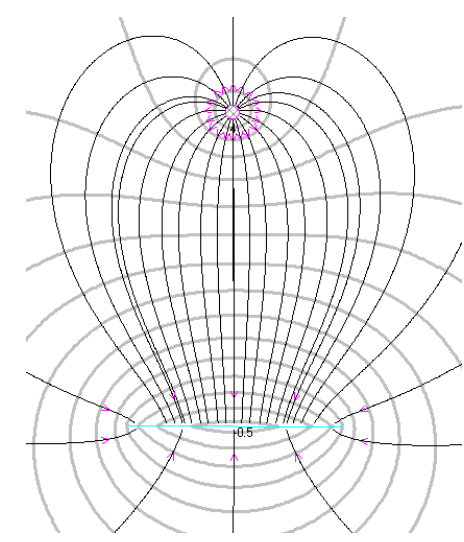

Fig. 6. Field lines (in thin black) and lines of constant potential (in thick gray), in a two dimensional topology consisting of a singular positive charge and a uniform distribution of negative charge, of equal total magnitude, along a linear segment.

definition of the potential as the negative gradient of the field, these are always perpendicular to the field lines.

By Theorem 1, the above figure also has an interpretation in the context of wireless sensor networks. In particular, the field lines show the optimal packet trajectories in an environment in which there are 5 singular traffic sources of equal magnitude, and a single, singular traffic sink at the center, collecting all the created information. The number of hops that a packet must make to go from an upstream location $A$ to a downstream location $B$ is proportional to the number of the lines of constant potential that the packet will cross. As there is a convergence of field lines to the central traffic sink, more nodes will be placed there, in order to support the traffic.

As a second example, in Fig. 6 we plot the field lines and lines of constant potential in a topology consisting of a singular positive charge, and a distribution of negative charge, of equal total magnitude, along a horizontal linear segment. The field lines of the figure are also the trajectories of packets in a topology where the positive and the negative charges are substituted with a traffic source and a traffic sink respectively. It is interesting to note that the optimal distribution of nodes actually implies that some of the packets will have to take very long routes, some of them actually arriving at the lower side of the traffic sink.

\section{EXTENSIONS}

\section{A. Traffic sources and sinks with limited mobility}

Until now, we have assumed that each location of infinitesimal size $(x, y)$ in the environment of Fig. 1 is associated with a fixed rate of information creation (or absorption). However, there are cases where it is best to associate whole regions of finite (and possibly large) area with a certain rate of information creation (or absorption), without specifying how this information rate should be distributed on the surface.

As an example, let us consider a sensor network designed to monitor the levels of humidity and temperature of a large plantation, and forward the measurements to a large central 
building. If we assume that a large number of wireless receivers, connected over very high capacity wired links with a central traffic sink, are placed along the circumference of the building, then the sensor network should be free to select which parts of the circumference of the building should receive how much traffic, in a way that minimizes the number of wireless nodes that must be deployed.

We model such environments by an information rate function $\rho(x, y)$, defined as in Section II, and a set of $t$ traffic regions $\left\{T_{i}\right\}$, where $i=1, \ldots, t$, each associated with an information rate $Q_{i}$, measured in bps. When $Q_{i}>0$, the region is creating information with rate $Q_{i}$, and when $Q_{i}<0$, it is absorbing information with rate $Q_{i}<0$. With no loss of generality, we assume that $\rho=0$ within each of the $t$ traffic regions. Equation (1) is modified as follows:

$$
\int \rho(x, y) d S+\sum_{i=1}^{t} Q_{i}=0
$$

where the surface integral is taken over the whole plane.

For any distribution $\mathcal{D}$ of the information rates $\left\{Q_{i}\right\}$ along the traffic regions $\left\{T_{i}\right\}$, there is an optimal node distribution, $d_{\mathcal{D}}(x, y)$, given by Theorem 1 , that minimizes the number of wireless nodes needed to support the traffic. A problem that arises naturally, is to find the optimal distribution $\mathcal{D}_{\text {opt }}$ of the rates $\left\{Q_{i}\right\}$ along the regions $\left\{T_{i}\right\}$, whose optimal node distribution $d_{\mathcal{D}_{\text {opt }}}(x, y)$ needs the minimum number $N$ of sensor nodes. In other words, we have a problem that consists of two consecutive minimizations.

We also note that

$$
N=\int d(x, y) d S=\frac{1}{K^{2}} \int|\mathbf{T}(x, y)|^{2} d S,
$$

therefore the minimization of nodes is equivalent to the minimization of the integral of the square of the magnitude of the traffic flow.

To solve this double minimization problem, let us consider its Electrostatics analogy: We have a setting with a fixed spatial electric charge density $\rho(x, y)$, and a set of regions $\left\{T_{i}\right\}$ on which we have placed a set of charges $\left\{Q_{i}\right\}$. Our assumption that the charges $Q_{i}$ can move everywhere along their corresponding regions $T_{i}$ means that, in the Electrostatics setting, these regions become conductors. As the traffic flow is mapped to the electric field, by (17), the equivalent problem becomes the calculation of the distribution of electric charge on a set of surfaces, such that the energy of the electric field, $\int|\mathbf{E}|^{2} d S$, is minimized.

Happily, this is exactly the same problem that nature solves when placing charges on conductors. In particular, Thomson's theorem ${ }^{7}$ [24], [28] states that charges placed on conductors distribute themselves so that the energy of the electric field is minimized.

This field can be determined by solving the equations:

$$
\nabla \cdot \mathbf{E}=\rho, \nabla \times \mathbf{E}=0,\left.\mathbf{E}\right|_{\infty}=\mathbf{0},
$$

\footnotetext{
${ }^{7}$ Named after William Thomson, better known as Lord Kelvin.
}

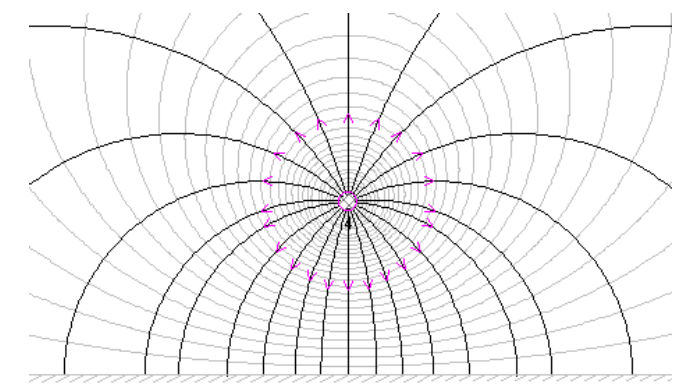

Fig. 7. Field lines (in thin black) and constant potential lines (in thick gray) when a singular positive charge is placed over a infinite conducting plane, infused with a negative charge of the same magnitude.

with the additional boundary condition:

$$
\mathbf{E}(x, y) \cdot \mathbf{t}=0,
$$

where $(x, y)$ is any point on the boundary $\partial T_{i}$ of a region $T_{i}$, and $\mathbf{t}$ is the unit vector parallel to the boundary $\partial T_{i}$ at point $(x, y)$, and the conditions (that follow from Gauss's law):

$$
\oint_{\partial T_{i}}[\mathbf{E} \cdot \hat{\mathbf{n}}] d l=Q_{i}, \quad \forall i=1, \ldots, m,
$$

where the path integral above is taken along the boundary $\partial T_{i}$ of the region $T_{i}$, and $\hat{\mathbf{n}}$ is a unit vector, normal at each point of the boundary, and pointing outwards.

Going back to sensor networks, it follows that the optimal distribution of traffic sources should create a traffic flow similar to the electric field induced by a placement of charges on a set of conductors, and this field $\mathbf{T}$ can be calculated by solving (18), (19), and (20), substituting $\mathbf{E}$ with $\mathbf{T}$, and taking $\rho$ to be the information density function and the $Q_{i}$ to be information rates.

As an example, in Fig. 7, we have placed a positive electric charge over a planar conductor infused with a negative electric charge of the opposite magnitude. In the figure, we have plotted the electric field, which can be calculated easily for this topology by the method of images [24]. We have also plotted lines of constant potential. It follows by the boundary condition (19) that the electric field is vertical to the conducting plane, therefore the potential (which is defined as the negative of its gradient) must be constant along the plane.

The above figure has a dual interpretation in the context of wireless sensor networks. In particular, it shows the optimal routes that packets must follow when moving from a singular source of information to a planar traffic sink, when there is no particular restriction on how many packets should be received at each location on the boundary of the sink. As the hop function is constant on the surface of the sink, it follows that the number of hops that a packet must make does not depend on the particular trajectory it decides to take. 


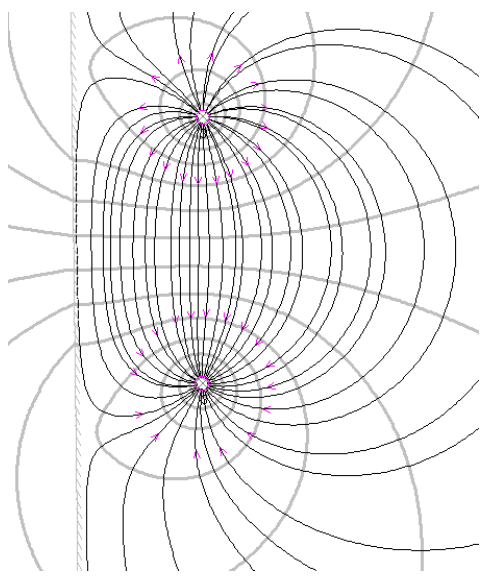

Fig. 8. Field lines (in thin black) and constant potential lines (in thick gray) created by a positive and a negative charge of equal magnitude, that are placed close to a boundary on which we impose the Neumann boundary condition $\mathbf{E}(x, y) \cdot \hat{\mathbf{n}}=0$.

\section{B. Forbidden regions}

Until now, we have assumed that we are free to place nodes anywhere on the plane. However, in many applications, the environment contains areas that are inaccessible to nodes. As an example, let us consider a large sensor network designed to monitor temperature and pressure readings in a large area that contains large bodies of water, such as rivers and lakes. As a second example, we may have a situation in which both the traffic sources and the traffic sinks are placed in a large room, and we are required to place all our wireless nodes on the floor of the room.

This situation can be modeled by assuming the existence of a set of forbidden regions $\left\{F_{i}\right\}$, where $i=1, \ldots, f$, such that through their boundaries no traffic can enter. Therefore, we require that:

$$
\nabla H \cdot \hat{\mathbf{n}}=\mathbf{T}(x, y) \cdot \hat{\mathbf{n}}=0,
$$

for any point $(x, y)$ along the boundary $\partial F_{i}$ of any forbidden region $F_{i}$. The unit vector $\hat{\mathbf{n}}$ is normal to the boundary $\partial F_{i}$ at the point $(x, y)$. The traffic function will be determined by solving the equations (13), together with the boundary condition (21), and perhaps the boundary conditions (19) and (20) if the topology also contains traffic regions.

Boundary conditions on scalar functions $H$ of the form of (21) are called Neumann's boundary conditions, and appear often in problems of Electrostatics. In the general form of Neumann's boundary conditions, the right hand side is an arbitrary function on the boundary of $F_{i}$. As an example, consider a material of some shape, in the interior of which the electric field is assumed zero, and on the boundary of which we have placed electric charge with a density $\sigma$. It follows from Gauss's law that the potential function $U$ should satisfy the Neumann boundary condition

$$
\nabla U \cdot \hat{\mathbf{n}}=-\mathbf{E}(x, y) \cdot \hat{\mathbf{n}}=-\sigma,
$$

for any point of the boundary of the material.

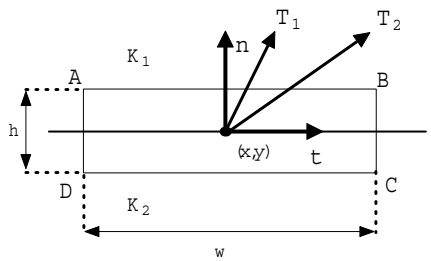

Fig. 9. The boundary between two regions with different node capabilities.

As an example, in Fig. 8 we plot the electric field that is created by two electric charges, of the same magnitude but of opposite sign, that are placed close to a planar boundary on which we impose the Neumann boundary condition $\mathbf{E}(x, y)$. $\hat{\mathbf{n}}=0$. The plotted field lines can also be interpreted as the optimal trajectories of packets when we place a singular traffic source and a singular traffic sink, of the same magnitude, close to a region in which no sensors may be placed.

\section{Nonhomogeneous environments}

Until now it has been assumed that all parts of the wireless network are equally efficient. This is a reasonable assumption when the network consists of a set of identical nodes, and in addition all parts of the environment present a similar challenge to the network. However, these assumptions may not always hold. For example, perhaps part of the sensor network lies close to a strong interferer that makes the communication harder. Alternatively, part of the network may be in an environment with heavy vegetation, which increases the attenuation of the signals with distance, or parts of the bandwidth are not available everywhere.

Such cases can be modeled by assuming that the coefficient $K$ appearing in (3) of Assumption 1 is no longer a constant, but is a function of the location. In particular, we modify Assumption 1 as follows:

Assumption 2: The environment is partitioned in a number of propagation regions $P_{i}$, where $i=1, \ldots, p$, each associated with a coefficient $K_{i}$, such that within $P_{i}$ we have:

$$
|\boldsymbol{T}(x, y)| \leq|\boldsymbol{T}(x, y)|_{\max }=K_{i} \sqrt{d(x, y)} .
$$

In this setting, equations (13) continue to hold within each region $P_{i}$. However, they break down at their boundaries, therefore they are not enough to uniquely specify the traffic flow. For this, we also need boundary conditions, that hold on the boundaries between any two different regions $P_{i}$ and $P_{j}$.

Let us concentrate, with no loss of generality, at a point $(x, y)$ on the boundary of regions $P_{1}$ and $P_{2}$. As shown in Fig. 9, let $\hat{\mathbf{n}}$ and $\hat{\mathbf{t}}$ be respectively the normal and tangential unit vector of the boundary at $(x, y)$. Also, let $\mathbf{T}_{1}$ and $\mathbf{T}_{2}$ be the traffic flows at point $(x, y)$, at the two sides of the boundary, which we decompose as follows:

$$
\mathbf{T}_{1}(x, y)=T_{n 1} \hat{\mathbf{n}}+T_{t 1} \hat{\mathbf{t}}, \quad \mathbf{T}_{2}(x, y)=T_{n 2} \hat{\mathbf{n}}+T_{t 2} \hat{\mathbf{t}} .
$$

Let us apply (4) on the perpendicular region $A B C D$, shown in Fig. 9, centered at $(x, y)$ and with height $h$ and width $w$. 
By taking first $h \rightarrow 0$, (4) becomes:

$$
\oint_{\partial A}[\mathbf{T}(x, y) \cdot \hat{\mathbf{n}}] d l=0 .
$$

By assuming $\mathbf{T}$ to be continuous on each side of the boundary, we have that

$$
\oint_{\partial A}[\mathbf{T}(x, y) \cdot \hat{\mathbf{n}}] d l=\left[T_{n 1}(x, y)-T_{n 2}(x, y)\right] w+o(w),
$$

and by dividing by $w$ and taking $w \rightarrow 0$, we have that:

$$
T_{n 1}=T_{n 2} \text {. }
$$

Next, we develop a boundary condition on the tangential components of the traffic flow. For this, let us consider the two streams of traffic moving tangentially along either side of the boundary. Since the traffic is optimally distributed, i.e., it uses the minimum number of wireless nodes, it follows that the moving of a part of the tangential traffic of the one side, to the other side, can only result to a net increase of the number of nodes needed. Therefore, we must have:

$$
\frac{\partial T_{t 1}}{\partial d_{1}}=\frac{\partial T_{t 2}}{\partial d_{2}},
$$

which, after noting that $T_{n 1}^{2}+T_{t 1}^{2}=K_{1}^{2} d_{1}$ and $T_{n 2}^{2}+T_{t 2}^{2}=$ $K_{2}^{2} d_{2}$, and some straightforward algebra, becomes:

$$
\left(K_{2}^{2}\right) T_{t 1}=\left(K_{1}^{2}\right) T_{t 2} \text {. }
$$

To summarize, the traffic flow must satisfy the partial differential equations (13) inside the propagation regions $P_{i}$, and the boundary conditions (25) and (26) at their boundaries. Contrary to the previous cases, this set of equations is not satisfied by the electric field of any setting in Electrostatics (for example, the tangential components of the electric field across a boundary must always be equal, by applying the integral form of Faraday's law [28]), However, it happens to be the set of equations that must be satisfied by the electric displacement vector $\mathbf{D}$ that would exist if $\rho$ were the density of free electric charge, and the regions $P_{i}$ contained dielectrics characterized by relative permittivities $\epsilon_{r}^{i}=K_{i}^{2}$. The electric displacement vector and the electric field are connected by the simple equation $\mathbf{D}=\epsilon_{r}^{i} \epsilon_{0} \mathbf{E}$, where $\epsilon_{0}$ is the permittivity of free space [28]. In other words, this sensor network problem can also be cast as an Electrostatics problem, with the minor differentiation that the traffic flow does not resemble the electric field, but rather the electric displacement.

As an example, in Fig. 10 we plot the lines of the electric field and lines of constant potential that are created by a distribution of charge similar to that of Fig. 6, but where in addition we assume that the lower half of the place is occupied by a dielectric with relative permittivity $e_{r}=10$, and the upper half of the plane is empty. The electric displacement vector is also parallel to the lines of the field, and its value in $P_{i}$ can be retrieved by the electric field by multiplying it with $\epsilon_{r}^{i} \epsilon_{0}$. In the context of wireless sensor networks, Fig. 10 shows how the traffic flow of the network of Fig. 6 would be modified if the lower half of the plane was substituted by nodes whose coefficient $K$ was increased by a factor of $\sqrt{10}$ (for example by increasing their available bandwidth by the same factor).

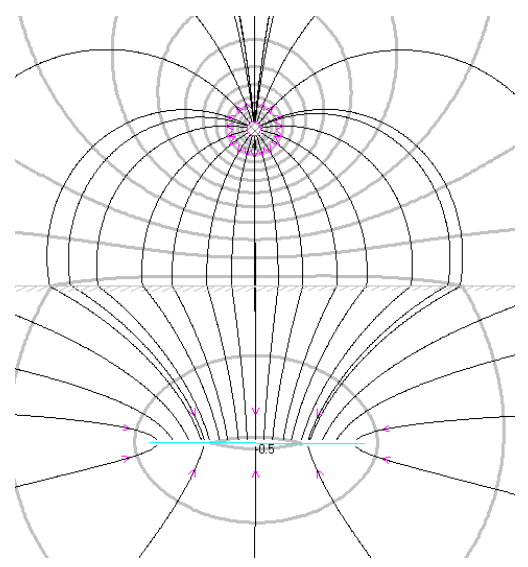

Fig. 10. The setting of Fig. 10, in which we have placed in the lower half of the plane a dielectric with relative permittivity equal to $\epsilon_{r}=10$.

\section{LimitaTiOnS}

In this section, we consider three cases in which our analogy between Electrostatics and wireless sensor networks breaks down. Because of space limitations, the discussion is brief, and more details can be found in [26].

\section{A. Three dimensional networks}

Until now, it was assumed that our sensor network is placed on a two dimensional plane. Let us now move to the study of three dimensional sensor networks. Such networks could be deployed, for example, in large buildings or in the sea.

The information density function, the node density function, and the traffic flow function can be defined similarly to their two dimensional equivalents, and they will now be measured in $\frac{\mathrm{bps}}{\mathrm{m}^{3}}, \frac{\text { nodes }}{m^{3}}$ and $\frac{\mathrm{bps}}{m^{2}}$ respectively. Equation (8) continues to hold, but using the three dimensional divergence operator [23]. Its physical interpretation, i.e., the conservation of information, remains unaltered.

As in the two dimensional case, we need an assumption linking the node density and the traffic flow function. One might be tempted to use Assumption 1 with no modification. However, a moment's reflection shows that its analogous in the three dimensions is the following:

Assumption 3: A location $(x, y, z)$, where the node density is $d(x, y, z)$, can support any traffic flow vector with a magnitude less or equal to a bound $|\boldsymbol{T}(x, y)|_{\max }$ which is proportional to the $\frac{2}{3}$ power of the density:

$$
|\boldsymbol{T}(x, y, z)| \leq|\boldsymbol{T}(x, y, z)|_{\max }=K^{\prime}[d(x, y, z)]^{\frac{2}{3}},
$$

where $K^{\prime}$ is a normalizing coefficient measured in bps.

To motivate (27), let us consider the three dimensional equivalent of the network of Fig. 2, which we plot in Fig. 11. In the figure, $\mathrm{m}^{3}$ nodes are arranged in a three dimensional regular grid. Assuming the same MAC protocol and physical layer as in the network of Fig. 2, we find that it is possible for the nodes to use a time division that consists of three slots, such that each node transmits during exactly one of the slots, to the node directly on its right. Using this time division, we 


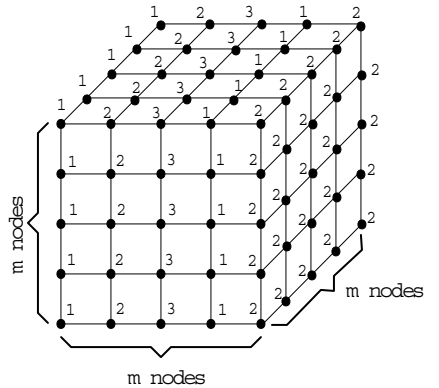

Fig. 11. A wireless three dimensional network of $m^{3}$ nodes placed on a regular grid. Nodes connected by edges can interfere with each other. A time division scheme exists that allows each node to transmit a packet to the node directly on the right, in one out of every three slots.

can create $m^{2}$ disjoint routes, each consisting of a set of $m$ nodes and being able to move traffic from left to right with a rate of $\frac{W}{3}$ bps. Therefore, an aggregate traffic stream of size $m^{2} \frac{W}{3}$ bps can be supported by this network of $m^{3}$ nodes. Working as in Section II, we arrive at (27).

As additional justification, we also note that in [29] the authors calculate the capacity of a three dimensional network, under a more realistic model for interference, and assuming a random placement of $n$ nodes. Their main result is that the aggregate throughput that the nodes can sustain is in the order of $\left[\frac{n}{\log n}\right]^{\frac{2}{3}}$. Assumption 3 may be viewed as the macroscopic version of that result, ignoring the logarithm that appears in the denominator, and the fact that the traffic of [29] is not going through the network, but originates and ends in the network.

Our problem remains the minimization of the number of nodes needed to support the traffic flow function, subject to the divergence constraint (8). In light of Assumption 3, we can formulate it as follows:

$$
\begin{array}{cc}
\text { minimize: } & \int|\mathbf{T}|^{b} d V, \\
\text { subject to: } & \nabla \cdot \mathbf{T}=\rho,\left.\quad \mathbf{T}\right|_{\infty}=0,
\end{array}
$$

where $b=\frac{3}{2}$. Note that we solved the same problem in the two dimensional case, but with $b=2$. Could it be that the solution $\mathbf{T}_{0}$ of (28) happens to be irrotational? Such a function would be unique, by the three dimensional version of Helmholtz's theorem. In general, the answer is no. An indirect proof of this, is by noting that the unique irrotational function that satisfies the constraints of (28), also minimizes the integral when $b=2$. The proof of this fact is a straightforward extension of the proof of Section III-B, in the three dimensions. It is intuitively clear than in the general case, the same function can not be the solution of both these optimization problems, and since there is only one irrotational function that satisfied the constraints, it follows that in general the solution of (28) will not be irrotational.

\section{B. Ultra WideBand communication}

Let us return to the consideration of two dimensional networks. Assumption 1 makes sense in networks in which bandwidth is limited, such as the ones considered in [20]. If, however, bandwidth is unlimited, as is in the case of Ultra WideBand communication, Assumption 1 should be modified.

The authors of [14] consider a network in which signal power decays polynomially with an exponent $\alpha>0$, and either $(i)$ the bandwidth available to each node is very large, or, equivalently, (ii), the maximum power $P$ available to each node is very small. In this setting, it is shown that the maximum aggregate throughput that a network of $n$ nodes can support is on the order of $n^{\frac{\alpha+1}{2}} \mathrm{bps}$, ignoring poly-logarithmic factors. Assuming that the network occupies a square of area $\epsilon^{2}$, so that the node density $d$ satisfies $n=\epsilon^{2} d$, we have that the through traffic that the network can support is on the order of $T \sim \epsilon d^{\frac{\alpha+1}{2}}$.

Working as in Section II-B, we derive the following assumption, that should be used in place of Assumption 1:

Assumption 4: A location $(x, y)$, where the node density is $d(x, y)$, can support any traffic flow vector with a magnitude less or equal to a bound $|\boldsymbol{T}(x, y)|_{\max }$ which is proportional to the $\frac{\alpha+1}{2}$ power of the density:

$$
|\boldsymbol{T}(x, y)| \leq|\boldsymbol{T}(x, y)|_{\max }=K d(x, y)^{\frac{\alpha+1}{2}},
$$

where $K$ is a normalizing coefficient measured in bps $\times m^{\alpha}$.

The new optimization problem becomes:

$$
\begin{array}{lc}
\text { minimize: } & \int|\mathbf{T}|^{\frac{2}{\alpha+1}} d S, \\
\text { subject to: } & \nabla \cdot \mathbf{T}=\rho,\left.\quad \mathbf{T}\right|_{\infty}=0 .
\end{array}
$$

By using the same line of reasoning as in Section $\mathrm{V}-\mathrm{A}$, we find that the solution of (29) will not necessarily be irrotational.

\section{Networks with multiple traffic types}

Until now, it was assumed that there is only one type of traffic in the network. Therefore, if more than one traffic stream flows through a point in the network, we are allowed to perform vector addition, and abstract the flow of traffic by a single vector, the traffic flow function at that point.

If, however, there are $m>1$ different types of traffic, each of them will have to be associated with its own traffic flow function $\mathbf{T}_{i}$, and its own information density function $\rho_{i}$, for which we will have:

$$
\nabla \cdot \mathbf{T}_{i}=\rho_{i}, i=1, \ldots, m .
$$

A point in the network through which different types of traffic cross, will have to divide its resources (in the time and/or the frequency domain) to support the different traffic types, therefore Assumption 1 will have to be substituted with the following:

Assumption 5: A location $(x, y)$, where the node density is $d(x, y)$, can support any combination of traffic traffic flow vectors $\boldsymbol{T}_{1}, \ldots, \boldsymbol{T}_{m}$, provided the following inequality holds:

$$
\sum_{i=1}^{m}\left|\boldsymbol{T}_{i}(x, y)\right| \leq|\boldsymbol{T}(x, y)|_{\max }=K \sqrt{d(x, y)}
$$

where $K$ is a normalizing coefficient measured in bps. 
Our new optimization problem is now the following:

$$
\begin{array}{cc}
\text { minimize: } & \int\left[\sum_{i=1}^{m}\left|\mathbf{T}_{i}\right|\right]^{2} d S, \\
\text { subject to: } & \nabla \cdot \mathbf{T}_{i}=\rho_{i},\left.\quad \mathbf{T}_{i}\right|_{\infty}=0, \quad \forall i=1, \ldots, m .
\end{array}
$$

As there is only one type of charge in Electrostatics, and many types of traffic in our settings, it is clear that our analogy can not be extended in any obvious way.

\section{CONCLUSIONS}

Our setting is a two dimensional environment, in which a distributed set of traffic sources is creating information that must be received by a distributed set of traffic sinks. The transport of information is handled by a network of wireless nodes, and our problem is the calculation of the optimal placement of nodes, such that the resulting network can transport all the created information while requiring the minimum number of nodes. We assume that we have so many nodes, that is does not make sense to specify their placement in terms of the positions of individual nodes, but in terms of the node density at any location of the environment.

Under a reasonable assumption, that approximates well the capabilities of many MAC and physical layers, we show that the optimal placement of nodes induces a traffic flow that resembles the electrostatic field that would exist if we substituted the traffic sources and sinks with positive and negative electric charges.

The analogy between sensor networks and Electrostatics can be extended in a number of different ways. For example, networks where we have limited freedom on the initial placement of sources and destinations can be mapped to Electrostatics topologies than include appropriately placed conductors. Also, environments that contain areas that are inaccessible to wireless nodes are similar to Electrostatics topologies that contain regions on whose boundaries we impose Neumann boundary conditions. Finally, environments that are composed of regions in which the capabilities of nodes differ are analogous to Electrostatics topologies in which there are placed dielectrics with different relative permittivities.

On the other hand, the analogy has important limitations. For example, if we move to the three dimensions, appropriately adapting our assumption on the physical and MAC layers, the optimal traffic flow is not in general irrotational, and so can not be mapped to any electrostatics field. The optimal traffic flow is also not irrotational if we consider an alternative assumption on the physical and MAC layers, that is more appropriate if the communication is power limited or the available bandwidth is infinite. Finally, the analogy can not be extended to include networks in which there is more than one type of traffic.

\section{REFERENCES}

[1] I. F. Akyildiz, W. Su, Y. Sankarasubramanian, and E. Cayirci, "A survey on sensor networks," IEEE Commun. Mag., vol. 40, no. 8, pp. 102-114, August 2002.

[2] E. Shih, S-H Cho, N. Ickes, R. Min, A. Sinha, A. Wang, and A. Chadrakasan, "Physical layer driven protocol and algorithm design for energy-efficient wireless sensor networks," in Proc. ACM MobiCOM, Rome, Italy, July 2001, pp. 272-287.
[3] J. Barros and S. D. Servetto, "The sensor reachback problem," submitted to the IEEE Trans. on Inform. Theory.

[4] A. Woo and D. Culler, "A transmission control scheme for media access in sensor networks," in Proc. ACM MobiCOM, Rome, Italy, July 2001, pp. 221-235.

[5] R. Verdone, "An energy-efficient communication protocol for a network of uniformly distributed sensors polled by a wireless transceiver," in Proc. IEEE ICC, Paris, France, June 2004.

[6] C. Intanagonwiwat, R. Govindan, and D. Estrin, "Directed diffusion: A scalable and robust communication paradigm for sensor networks," in Proc. ACM MobiCOM, Boston, MA, August 2000, pp. 56-67.

[7] C. Shen, C. Srisathapornphat, and C. Jaikaeo, "Sensor information networking and applications," IEEE Pers. Commun., vol. 8, no. 4, pp. 52-59, Aug. 2001.

[8] A. Ephremides, "Energy concerns in wireless networks," IEEE Wireless Commun. Mag., vol. 9, no. 4, pp. 48-59, Aug. 2002.

[9] R. Iyer and L. Kleinrock, "QoS control for sensor networks," in Proc. IEEE ICC, Anchorage, AL, May 2003, pp. 517-521.

[10] T. Ajdler, R. Cristescu, P. L. Dragotti, M. Gastpar, I. Maravic, and M. Vetterli, "Distributed signal processing and communications: On the interactions of sources and channels," in Proc. IEEE ICASSP, Hong Kong, China, April 2003, vol. 4, pp. 852-855.

[11] S. D. Servetto, "Distributed signal processing algorithms for the sensor broadcast problem," in Proc. Conference on Information Sciences and Systems, Baltimore, MD, Mar. 2003.

[12] P. Jacquet, "Geometry of information propagation in massively dense ad hoc networks," in Proc. ACM MobiHOC, Roppongi Hills, Japan, May 2004, pp. 157-162.

[13] S. Bandyopadhyay and E. J. Coyle, "Spatio-temporal sampling rates and energy efficiency in wireless sensor networks," in Proc. IEEE INFOCOM, Hong Kong, China, Mar. 2004, vol. 2, pp. 976-985.

[14] R. Negi and A. Rajeswaran, "Capacity of power constrained ad-hoc networks," in Proc. IEEE INFOCOM, Hong Kong, China, Mar. 2004.

[15] A. Howard, M. J. Matarić, and G. S. Sukhatme, "Mobile sensor network deployment using potential fields: A distributed, scalable solution to the area coverage problem," in Proc. Inter. Symp. on Distributed Autonomous Robotics Systems, Fukuoka, Japan, June 2002, pp. 299308.

[16] M. Kalantari and M. Shayman, "Routing in wireless ad hoc networks by analogy to electrostatic theory," in Proc. IEEE ICC, Paris, France, June 2004.

[17] M. Kalantari and M. Shayman, "Energy efficient routing in wireless sensor networks," in Proc. Conference on Information Sciences and Systems, Princeton University, NJ, Mar. 2004.

[18] N. T. Nguyen, A.-I A. Wang, P. Reiher, and G. Kuenning, "Electric-fieldbased routing: A reliable framework for routing in MANETs," ACM Mobile Computing and Communications Review, vol. 8, no. 1, pp. 3549, Apr. 2004.

[19] J. Silvester and L. Kleinrock, "On the capacity of multihop slotted ALOHA networks with regular structures," IEEE Trans. Commun., vol. 31, no. 8, pp. 974-982, Aug. 1983.

[20] P. Gupta and P. R. Kumar, "The capacity of wireless networks," IEEE Trans. Inform. Theory, vol. 46, no. 2, pp. 388-404, Mar. 2000.

[21] M. Franceschetti, O. Dousse, D. N. C. Tse, and P. Thiran, "Closing the gap in the capacity of random wireless networks," in Proc. IEEE ISIT, Chicago,, Mar. 2003.

[22] E. W. Weisstein, "Path integral," From MathWorld - A Wolfram Web Resource, mathworld.wolfram.com/PathIntegral.html.

[23] H. M. Schey, Div, Grad, Curl, and All That: An Informal Text on Vector Calculus, W. W. Norton and Company, 3rd edition, Oct. 1996.

[24] J. D. Jackson, Classical Electrodynamics, Wiley Text Books, 3rd edition, 1998.

[25] E. W. Weisstein, "Path integral," From MathWorld — A Wolfram Web Resource, mathworld.wolfram.com/LineIntegral .html.

[26] S. Toumpis and L. Tassiulas, "On the efficient spatial distribution of nodes in massively dense sensor networks," in preparation for submission to journal publication.

[27] N. Bigdely, "Equipotential surfaces and E lines," Available online at www.electrostatics3d.com.

[28] W. K. H. Panofsky and M. N. Phillips, Classical Electricity and Magnetism, Reading, MA: Addison-Wesley Publications, 1955.

[29] P. Gupta and P. R. Kumar, "Internets in the sky: The capacity of three dimensional wireless networks," Communication in Information and Systems, vol. 1, no. 1, pp. 33-49, Jan. 2001. 